\title{
A BASIC STEP TOWARD THE CO-LINE TULLY-FISHER RELATION
}

\author{
YOSHIAKI SOFUE AND FRANZ SCHÖNIGER \\ Institute of Astronomy, The Universtity of Tokyo, Mitaka, Tokyo 181
}

\section{INTRODUCTION}

The Tully-Fisher relation makes use of the correlation between the HI linewidth and the luminosity of a galaxy and is one of the most powerful tools to estimate distances of galaxies. Because of the smaller beam the $\mathrm{CO}$ line might be an alternative to $\mathrm{HI}$ for distant galaxies which so far can not be examined even by the largest telescopes (Dickey and Kazes 1992).

In order to consider the question whether the $\mathrm{CO}$ lineprofile could be used as an alternative or a supplement for $\mathrm{HI}$ in the Tullly-Fisher relation, we compared the total $\mathrm{CO}(\mathrm{J}=2-1)$ line profiles of ten galaxies with the corresponding $\mathrm{HI}$ profiles. Four almost perfect edge-on galaxies (NGC 891, NGC 4565, NGC 4631 and NGC 3079) and the Seyfert galaxy NGC 1808 have been observed in CO using the Nobeyama $45-\mathrm{m}$ telescope. The HI profiles and the data of the other galaxies has been taken from the literature.

\section{RESULTS}

For the isolated edge-on galaxies NGC 891 and NGC 4565 we found an almost perfect coincidence between the profiles (HI profiles from Rots 1980). This can be understood by considering the rotation curves of these galaxies together with the radial distribution of the atomic and the molecular gas density. The atomic and the molecular gas densities peak at almost the same radius, and at this distance from the galactic center the rotation curve has already become flat. Therefore the horns of the line profiles occur at the same radial velocity.

The galaxies NGC 4631 (HI profile from Rots 1980) and IC 342 (CO data from Young and Scoville 1982, HI profile taken from Rots) both show a broader $\mathrm{HI}$ than $\mathrm{CO}$ profile and a blue-shifted systemic celocity of HI compared with CO. NGC 4631 is a peculiar and interacting galaxy. The broader HI profile as well as the blue-shifted systemic velocity of $\mathrm{HI}$ might be due to tidal disturbance on the HI envelope (Weliachew et al. 1978). This might also be the reason of the broader HI profile in the case of IC 342 (Newton 1980).

Because $\mathrm{CO}$ is more concentrated to the center of a galaxy than HI, it's not as sensitive to tidal distortions as HI. This implies, that the CO Tully-Fisher relation is more reliable for interacting galaxies.

In the case of the peculiar galaxies NGC 1068 (CO from Scoville et al. 1983, HI from Staveley-Smith and Davies 1987), NGC 1808 (HI from Reif et al. 1982) and NGC 3079 (HI from Irwin and Seaquist 1991) the CO profile is broader than that of $\mathrm{HI}$. 



FIGURE I $\mathrm{CO}$ and HI profiles for our sample of galaxies. Rotation curves and density distributions for NGC 891 and NGC 4665 and a plot of CO vs HI linewidth is also shown.

A good agreement was found for NGC 6946 (CO from Young and Scoville 1982, HI from Rots 1980), M31 (Koper et al 1991), the quasar host galaxy I Zw 1 (CO from Barvainis and Alloin 1989, HI from Condon et al. 1985) and also for the bright IRAS galaxy NGC 7469 (CO from Young et al. 1986, HI from Mirabel 1982) despite the blue-shifted systemic velocity of $\mathrm{CO}$ in the case of NGC 7469.

Finally we stress that the $\mathrm{CO}$ line profile might be a promising alternative for HI not only for distant but also for nearby galaxies. A paper about this topic containing a complete list of references is in preparation by Sofue and Schöniger.

\section{REFERENCES}

Dickey, J., and Kazes, I. 1992, ApJ, 393, $530 .$.

Newton, K. 1980, MNRAS, 191, 169

Weliachew, L., Sancisi, R., and Guelin, M. 1978, $A \& A, 65,37$. 